\title{
El aprendizaje-servicio como vía para el desarrollo de competencias interculturales en la Universidad
}

\section{Service-learning as a way to develop intercultural competence in Higher Education}

\author{
Alexandre Sotelino Losada $^{1}$ \\ Alexandre.sotelino@usc.es \\ Miguel Ángel Santos Rego \\ Miguelangel.santos@usc.es \\ Jesús García Álvarez \\ Jesus.garcia.alvarez@usc.es \\ Universidade de Santiago de Compostela, España
}

\section{Resumen:}

El proyecto PEINAS (Pedagogía Intercultural y aprendizaje-servicio) nace en el curso 2010/2011 asociado a la asignatura rotulada como "Pedagogía Intercultural" (Segundo curso del Grado en Pedagogía, Universidade de Santiago de Compostela). El proyecto tiene por finalidad mejorar el conocimiento de la realidad social y educativa que viven cotidianamente las diferentes comunidades culturales en Galicia. Para ello se establecen vínculos con entidades en las que los estudiantes desarroIlan un servicio, sobre bases académicas, en función de necesidades específicas.

Lo que esta aportación pretende es mostrar el desarrollo de competencias cívicosociales en estudiantes universitarios que participan en un proyecto de estas características. Además, comparamos alumnos/

\begin{abstract}
:
The PEINAS project (Intercultural Pedagogy and Service-Learning) was born in the academic year 2010/2011 associated to an academic subject called "Intercultural Pedagogy" (Second year of the Degree in Pedagogy, University of Santiago de Compostela). The project aims to improve the knowledge of the different cultural communities in Galicia about their social and educational realities. Thus, students create links with different entities where they undertake a social service, on an academic basis, according to specific needs. What this paper intends is to explore the development of civic-social competence in university students who participate in a project of these characteristics. In addition, we compare students who participated in the project with those who did not
\end{abstract}

1 Dirección para correspondencia (correspondence address):

Alexandre Sotelino Losada. Departamento de Pedagogía y Didáctica. Grupo de Investigación Esculca. Red RIES de Investigación. Universidade de Santiago de Compostela. Facultad de Ciencias de la Educación. C/ Xosé María Suárez Nuñez. s/n. Campus Vida. 15782, Santiago de Compostela (España). 
El aprendizaje-servicio como vía para el desarrollo de competencias interculturales en la Universidad

Alexandre Sotelino Losada, Miguel Ángel Santos Rego y Jesús García Álvarez

as participantes y no participantes en la citada modalidad formativa, haciendo uso del postest elaborado en el marco de un proyecto del Plan Nacional de I+D+I (EDU2013-41687-R), lo cual nos ha de permitir ver las diferencias entre los grupos de estudiantes.

Terminamos señalando que los estudiantes que participan en experiencias de esta índole incrementan sus oportunidades de adquirir una serie de competencias de corte relacional y cívico-social, de apreciable importancia para su formación práctica y el desarrollo de estructuras sólidas de aprendizaje.

\section{Palabras clave:}

Aprendizaje-servicio; Educación Superior; Educación cívica; Pedagogía Intercultural. participate. To this end, we use a post-test elaborated within the framework of a project of the Spanish National Plan for Research (EDU2013-41687-R), which allows us to see the differences among groups of students. The conclusions highlight the fact that students who participate in such experiences increase their opportunities to acquire a series of relational and civicsocial skills of considerable importance for their practical training and the development of sound learning structures.

\section{Keywords:}

Service-learning; Higher education; Civic education; Intercultural Pedagogy.

\section{Résumé :}

Le projet Peinas (Pédagogie Interculturelle et apprentissage-service) a commencé pendant l'année scolaire 2010/2011 associé à la matière intitulée "Pédagogie Interculturelle" (deuxième année de diplôme universitaire en pédagogie, Université de Santiago de Compostela). Le but du projet est d'améliorer la connaissance de la réalité sociale et éducative que les différentes communautés culturelles vivent en Galice au quotidien. À cette fin, des liens sont établis avec des entités dans lesquelles les étudiants développent un service, sur une base académique, en fonction de besoins spécifiques.

Cette contribution vise à montrer le développement des compétences civico-sociales chez les étudiants universitaires qui participent à un projet de ces caractéristiques. De plus, nous comparons les étudiants participants et non participants au mode de formation cité, en utilisant le post-test élaboré dans le cadre d'un projet national (EDU201341687-R), qui doit nous permettre de voir les différences entre les groupes d'étudiants.

Nous concluons en soulignant que les étudiants qui participent à des expériences de cette nature augmentent leurs chances d'acquérir une série de compétences relationneIles et civico-sociales, d'une importance considérable pour leur formation pratique et le développement de solides structures d'apprentissage.

\section{Motsclés:}

Apprentissage par le service, Enseignementsupérieur, Éducationcivique; Pédagogieinterculturelle

Fecha de recepción: 1-6-2018

Fecha de aceptación: 15-10-2018 


\section{Introducción}

El artículo que presentamos a continuación pone su foco en la Pedagogía Intercultural como ámbito de estudio del currículum de los estudiantes de Pedagogía. Somos de los convencidos de que la idea de una sociedad positivamente intercultural aún no ha sido alcanzada, en lo cual han influido diversos factores de todo tipo: económicos, sociales, educativos, culturales... (Martinez-Usarralde, 2009; Santos-Rego, 2017). En este sentido la Pedagogía Intercultural puede, y debe, contribuir a mejorar, con efectividad y sentido de justicia, el sistema educativo y social, velando siempre por los intereses de todos, pero sin descentrar la atención de aquellos que corren más riesgos de exclusión (Santos-Rego y Lorenzo, 2012). Es por ello que aquellos docentes que nos dedicamos a la investigación en este ámbito tenemos que aportar estrategias que permitan seguir progresando en esta larga singladura que hemos emprendido, fijando la mirada en aquellos que deben sentar las bases de una sociedad más igualitaria e inclusiva, esto es, educadores y educadoras, pedagogos y pedagogas, que están egresando de nuestras universidades.

El aprendizaje-servicio (en adelante ApS) es una "metodología experiencial que aúna aprendizaje y servicio comunitario en un único proyecto con base académica y cívica" (Sotelino, Santos-Rego, y Lorenzo, 2016, p. 216). De este modo, se configura como una vía para seguir afianzando el avance de una educación más intercultural, a partir de una praxis social efectiva. Precisamente, lo que nos ocupa en este trabajo es presentar un proyecto de aprendizaje-servicio que nace en el año 2011, y que se desarrolla desde entonces en el marco de la asignatura 'Pedagogía Intercultural' del Grado en Pedagogía de la Universidad de Santiago de Compostela.

En la citada materia se había trabajado anteriormente con metodologías de corte cooperativo e implicando directamente al alumnado en su proceso de aprendizaje, pero la definición de este proyecto de ApS ha constituido una nueva apuesta por afianzar conocimientos desde una praxis social y aplicada a las realidades circundantes. Al mismo tiempo, y tomando como referencia el contexto americano, hemos podido conocer proyectos en esta línea que han inspirado la puesta en marcha y consolidación del PEINAS, siempre ajustado a nuestra realidad (Boyle-Baise, 1998; Einfeld y Collins, 2008; O’Grady, 2000; Santos-Rego, 2013).

Lo que se pretende con el proyecto PEINAS (Pedagogía Intercultural y 
aprendizaje-servicio) es que el alumnado de la citada materia, ubicada en segundo curso del grado, aprenda realizando un servicio en el marco de entidades que trabajan en la gestión de la diversidad cultural. Estas organizaciones son de diferente índole y se encuentran repartidas por todo el territorio gallego, por lo que el servicio se diversifica en función de necesidades concretas. De lo que se trata es de que el aprendizajeservicio pueda entenderse y aprovecharse como vector de innovación en la Pedagogía Intercultural, desde una mejor conexión entre lo que sucede en entidades con apreciable recorrido social y un contenido académico inserto en el Grado de Pedagogía.

La metodología que se utiliza en el desarrollo de esta experiencia es la denominada aprendizaje-servicio (service-learning). Se entiende como actividad académica con clara proyección social, siendo el objetivo que el alumnado adquiera los contenidos programados en el marco curricular de las diferentes materias, pero realizando un servicio de utilidad en el medio social (Lorenzo y Santos-Rego, 2009).

En lo que se refiere a las fases en las que se articula el Proyecto PEINAS, se observa una correspondencia significativa en relación a los pilares básicos que debe reunir toda experiencia de aprendizaje-servicio (Santos-Rego, Sotelino, y Lorenzo, 2015):

1. Preparación por parte del profesor. En esta fase se establecen objetivos de aprendizaje y se introduce al grupo en la dinámica de trabajo. Lo importante es conseguir que el alumnado entienda desde un principio la diferencia que se establece entre una dinámica de ApS y otro tipo de iniciativas, evitando que puedan surgir posibles problemas a posteriori.

2. Planificación con el grupo. En este momento se detectan las necesidades de partida, se establece un plan de trabajo y se define el proyecto. Se trata de una fase en la que podremos alcanzar grandes logros pedagógicos si conseguimos implicar al alumnado en la detección de las necesidades y en el desarrollo de un plan de acción. De este modo, el alumnado podría implicarse en el proyecto desde una perspectiva profesional, construyendo así una identidad técnica que irá en favor de una óptima consecución de metas académicas.

3. Ejecución con el grupo. Tras el trabajo inicial de planificación y diseño, se implementa el servicio acordado con cada una de las entidades participantes, al tiempo que se establecen sesiones de seguimiento para establecer vínculos académicos. 
4. Evaluación con el grupo. El proyecto tiene que suponer una evaluación continua del proceso, lo cual permite integrar los aprendizajes, adecuarlos al servicio y mejorar así la calidad de la experiencia (Villa y Poblete, 2007). La reflexión es el punto de unión, el vínculo entre el aprendizaje académico y el servicio (Goldberg y Coufal, 2009; Páez y Puig, 2013).

Las metas superadas durante el proceso forman parte sustancial del éxito del proyecto de ApS, en tanto que el alumnado mantenga la motivación para alcanzar un objetivo final con tintes técnico-profesionales (Martinez-Vivot y Folgueiras, 2015). No debemos dejar de hacer un seguimiento pormenorizado si lo que queremos saber es si realmente nuestra experiencia es de aprendizaje-servicio y no de otro tipo de metodología (Deeley, 2016; Driscoll, Holland, Gelmon y Kerrigan, 1996).

5. Evaluación del educador. Toda iniciativa de ApS debe tener una clara intencionalidad pedagógica, en tanto que proyecto educativo, planificado y evaluado por parte del profesor. Es por ello que una vez finalizada la experiencia hemos de realizar una evaluación de conjunto que atienda también al funcionamiento de las entidades, a las dinámicas de aprendizaje desarrolladas, al transcurso del servicio, al papel del docente, entre otros, y a todas aquellas variables que puedan contribuir a la mejora de la experiencia en futuras ediciones (Lorenzo, Mella, García, y Varela, 2017).

El número de estudiantes que ha participado en este proyecto de ApS ha variado en cada edición en función de la capacidad de acogida por parte de las diferentes entidades. De este modo, en la primera edición (curso 2010/2011) fueron 15 personas las que realizaron el servicio, aumentando posteriormente, de manera progresiva, hasta alcanzar los 25 alumnos/as implicados en la edición 2017/2018. La selección de estos estudiantes atiende a criterios de proximidad al lugar de servicio, disponibilidad y orden de inscripción. El perfil del alumnado implicado es variado, aunque es cierto que una buena parte de ellas, ya que en su mayoría son alumnas, no han participado en experiencias previas de servicio a la comunidad. Esto podría reforzar todavía más el impacto en su trayectoria personal y, por ende, profesional (Tejada, 2013). Los demás alumnos y alumnas que no pueden participar en la experiencia siguen la otra modalidad de evaluación, participando de diferentes sesiones in- 
El aprendizaje-servicio como vía para el desarrollo de competencias interculturales en la Universidad

Alexandre Sotelino losada, Miguel Ángel Santos Rego y Jesús García Álvarez

teractivas. La estancia en las diferentes entidades tiene lugar durante el tiempo de la propia asignatura, esto es, un cuatrimestre, entre los meses de enero y mayo (Priegue y Sotelino, 2016).

Por otro lado, la población destinataria con la que se trabaja es diversa, y depende de la propia naturaleza de las entidades que participan (ver tabla 1).

Tabla 1

Entidades participantes en el proyecto PEINAS

\begin{tabular}{ll}
\hline Entidades & Curso/s \\
\hline Asociación Plural.es (Caldas de Reis) & $\begin{array}{l}2010 / 2011 \text { (hasta } \\
\text { la actualidad) }\end{array}$ \\
\hline Cruz Roja (Santiago de Compostela) & $\begin{array}{l}2010 / 2011 \text { (hasta } \\
\text { la actualidad) }\end{array}$ \\
\hline ONG Equus Zebra (A Coruña) & $\begin{array}{l}2010 / 2011 \text { (hasta } \\
\text { la actualidad) }\end{array}$ \\
\hline AISO-Asociación de Inmigrantes Senegaleses de Ourense & $2010 / 2011$ (hasta \\
& $2015 / 2016)$ \\
\hline Cáritas (Santiago de Compostela) & $\begin{array}{l}2011 / 2012 \text { (hasta } \\
\text { la actualidad) }\end{array}$ \\
\hline Ecos do Sur (A Coruña) & $2011 / 2012$ \\
\hline Médicos do Mundo (Santiago de Compostela) & $2012 / 2013$ (hasta \\
& la actualidad) \\
\hline Fundación Juan Soñador - Proyecto Teranga (Ourense) & $2012 / 2013$ (hasta \\
& la actualidad) \\
\hline Fundación Secretariado Gitano (Lugo) & $2012 / 2013$ \\
\hline Fundación Juan Soñador - Proyecto Teranga (Vigo) & $2013 / 2014$ (hasta \\
& la actualidad) \\
\hline Cruz Roja (Ourense) & $2014 / 2015$ (hasta \\
& la actualidad) \\
\hline Cruz Roja (Pontevedra) & $2014 / 2015$ (hasta \\
& la actualidad) \\
\hline Proyecto Vagalume (Santiago de Compostela) & $2015 / 2016$ (hasta \\
& la actualidad) \\
\hline Fundación Secretariado Gitano (Pontevedra) & $2016 / 2017$ (hasta \\
& la actualidad) \\
\hline Humanismo y Democracia (Santiago de Compostela) & $2016 / 2017$ \\
\hline Fundación Ronsel (Santiago de Compostela) & $2017 / 2018$ \\
\hline Centro Sociocultural Aurelio Aguirre (Santiago de Com- & $2017 / 2018$ \\
postela) & \\
\hline
\end{tabular}


En este sentido, y de manera genérica, los servicios derivados de este proyecto se pueden agrupar del siguiente modo:

1. Inserción sociolaboral de colectivos inmigrantes. El alumnado estudia los perfiles profesionales de los usuarios de las diferentes entidades y posteriormente desarrolla una intervención adaptada. En ocasiones, los propios participantes han impartido diferentes cursos formativos referidos a habilidades sociorelacionales, elaboración de currículos, técnicas de búsqueda de empleo, manejo de plataformas online...

Algunas de las organizaciones en las que se viene desarrollando el servicio son: Cáritas (Santiago de Compostela), Cruz Roja (Santiago de Compostela, Pontevedra y Ourense), Ecos do Sur (A Coruña), Proyecto Vagalume (Santiago de Compostela), Humanismo y Democracia (Santiago de Compostela) y Fundación Ronsel (Santiago de Compostela).

2. Optimización del aprendizaje con personas de origen inmigrante. Las alumnas y alumnos preparan diferentes sesiones de técnicas de estudio con los usuarios para ayudarles a optimizar su proceso de aprendizaje. De manera eventual, también han participado en programas de alfabetización acompañando y apoyando al personal encargado de este tipo de programas.

Las entidades donde ha tenido lugar este servicio han sido: Plural.es (Caldas de Reis, Pontevedra), Equus Zebra (A Coruña), Fundación Juan Soñador (Ourense e Vigo), Secretariado Gitano (Lugo), Secretariado Gitano (Pontevedra) y Centro Sociocultural Aurelio Aguirre de Conxo (Santiago de Compostela).

3. Ocio y tiempo libre. Este programa se ha desarrollado preferentemente en la Asociación de Inmigrantes Senegaleses de Ourense (AISO), donde se han puesto en marcha diferentes iniciativas vinculadas a la pedagogía del ocio. Es así que el alumnado programaba múltiples actividades para los niños durante la duración del proyecto, ayudando en la organización de actividades relevantes, entre otras, la del Día de Senegal.

4. Difusión y sensibilización. Por último, en Médicos do Mundo (Santiago de Compostela) se han diseñado diferentes campañas de sensibilización, procurando empoderar a los receptores de las diferentes iniciativas en habilidades relacionales e interculturales. Así, se han llevado a cabo juegos de rol, dinámicas participativas 
El aprendizaje-servicio como vía para el desarrollo de competencias interculturales en la Universidad

Alexandre Sotelino Losada, Miguel Ángel Santos Rego y Jesús García Álvarez

y campañas, en la universidad y en institutos de Secundaria de zonas cercanas a la capital de Galicia. Todos ellos diseñados, programados e implementados por el alumnado participante.

Los aprendizajes que se extraen de esta experiencia podrían clasificarse en tres grupos. En primer lugar, estarían los directamente relacionados con la materia de Pedagogía Intercultural, esto es: concepto de pedagogía intercultural, intervención pedagógica en educación intercultural, técnicas y estrategias específicas (juegos de rol, aprendizaje cooperativo, tutoría entre iguales...) o conocimientos culturales, entre otros.

Pero en las experiencias de ApS se aprende mucho más que los contenidos teóricos específicos de la disciplina en que se ubican. En este sentido, el alumnado adquiere conocimientos y competencias de la titulación que cursan (aquí el Grado en Pedagogía), destacando: diseño e implementación de programas educativos, evaluación de las escuelas, instituciones y recursos educativos, programación e implementación de técnicas de estudio o habilidades didácticas y metodológicas.

Además, este tipo de iniciativas supone para el participante un incremento en su desarrollo personal, experiencia que incide en una mejora significativa del aprendizaje de contenidos y habilidades específicas derivadas de la práctica, junto a valores asociados. Produce un incremento de la motivación, la autoestima y las expectativas personales, ya que su acción tiene un valor tangible en un contexto real y concreto. Podríamos denominarlos, entonces, "aprendizajes para la vida" (Santos-Rego et al., 2015).

Para optimizar el proceso de aprendizaje se hace un seguimiento a base de sesiones grupales, en las que se fomenta la reflexión en torno al servicio que se realiza, sin descuidar en ningún momento el contenido de la materia, e incluso extendiendo el servicio a otras disciplinas del Grado (Sotelino, 2014). Tales sesiones tienen un sentido claro en pos de una evaluación continua y formativa, articulando procesos de intercambio y reciprocidad entre estudiantes y profesor, tan importantes para optimizar la reflexión crítica (Deeley, 2016).

En definitiva, creemos que el aprendizaje-servicio, metodología educativa que informa el proyecto PEINAS, ha devenido en estrategia de alto valor pedagógico para la formación de estudiantes universitarios, que en nuestro caso contribuye al desarrollo de capacidades en tanto que permite una mejor gestión de la diversidad cultural dentro y fuera de las 
escuelas. De este modo, el citado proyecto contribuye a despertar en el alumnado una mayor sensibilidad intercultural, ligada al desarrollo de pautas de convivencia susceptibles de reducir prejuicios, a partir del contacto y comunicación entre personas y grupos con diferentes características.

\section{El desarrollo de competencias cívico-sociales en los participantes del proyecto PEINAS}

Una vez que hemos aclarado de manera pormenorizada el desarrollo del proyecto PEINAS, así como justificado su efectiva consolidación en la realidad de la USC, lo que nos interesa es estudiar su potencialidad en relación a la adquisición de competencias cívico-sociales en el alumnado participante. En este sentido, y en el marco de la investigación "Aprendizaje-servicio e innovación en la Universidad. Un programa para la mejora del rendimiento académico y el capital social de los estudiantes" (EDU2013-41687-R; BOE 1/08/2014) hemos tenido la oportunidad de comprobar algunos de sus resultados. La investigación ha sido desarrollada por seis universidades españolas (UCM, UDC, UCO, UV, UNAV y USC), bajo la coordinación del GI-Esculca, de la Universidad de Santiago de Compostela.

Tal estudio se ha planteado en tres fases diferenciadas. Una inicial de carácter exploratorio para conocer las prácticas de ApS, o cercanas a la metodología, que se desarrollan en las universidades participantes y analizar las formas de organización de las tareas de aprendizaje por parte de los profesores universitarios (cultura docente) (Santos-Rego, Jover, Naval, Álvarez Castillo, Vázquez y Sotelino, 2017). La segunda de las etapas es el diseño de un programa de ApS en la Universidad y de los instrumentos y protocolos de evaluación del mismo. En esta segunda fase se realiza un proceso formativo y de acompañamiento con aquellos profesores/as que se interesen por el ApS. Y en una última fase, es donde se implementa y evalúa, según el diseño establecido anteriormente, cada uno de los proyectos de ApS (estudio experimental) (Lorenzo et al., 2017; Álvarez, Martínez, González, y Buenestado, 2017).

Precisamente, en estas dos últimas fases del proyecto es donde se enmarca el artículo que aquí se presenta. De lo que se trata, finalmente, es de reconocer y analizar el impacto que ha tenido el proyecto PEINAS en 
el desarrollo de competencias cívico-sociales, y por ende interculturales, en los alumnos/as participantes. A continuación, explicamos cómo se ha procedido a recoger y analizar los datos.

\subsection{Objetivos}

Como ya hemos mencionado, el objetivo del trabajo es analizar el impacto de la metodología aprendizaje-servicio en el desarrollo de competencias cívico-sociales en estudiantes universitarios.

\subsection{Participantes del estudio}

La muestra productora de datos está conformada por un total de 50 estudiantes, de los 63 matriculados en la asignatura Pedagogía Intercultural (curso 2016/2017) de segundo curso del Grado en Pedagogía de la USC.

Del total de estudiantes, el $42 \%$ de ellos, un conjunto de 21 sujetos, participan en el proyecto PEINAS conformando así el grupo experimental, mientras que el 58\%, optó por la otra modalidad de evaluación ofrecida, y por lo tanto servirá como grupo control.

\subsection{Instrumento de medida}

Como ya hemos señalado, este estudio se enmarca dentro de un proyecto de investigación más amplio, y en el que se han aplicado diferentes instrumentos. En este caso concreto utilizamos la escala de competencias cívico-sociales (21 ítems) que forma parte del Cuestionario sobre competencias cívico-sociales y autoeficacia del alumnado universitario (CUCOCSA). Esta se aplicó a ambos grupos una vez finalizado el proyecto.

La citada escala ha sido redactada valiéndonos del formato Likert, con cinco grados, donde el valor 5 implicaba el mayor grado de acuerdo y donde 1 era totalmente en desacuerdo. Cabe señalar que este cuestionario y, por supuesto, las escalas, han sido validadas convenientemente, y además han resultado fiables en las pruebas aplicadas.

\subsection{Variables}

Como variable independiente, o de agrupamiento, referimos el hecho de haber participado en el proyecto de aprendizaje-servicio. Y, a su vez, 
como variables dependientes analizaremos las respuestas que dan los estudiantes en la escala de competencias cívico-sociales del cuestionario CUCOCSA.

\subsection{Análisis de resultados}

A continuación, procedemos a presentar los principales resultados hallados a partir de la comparación entre los dos grupos (control y experimental). La decisión del carácter estadísticamente significativo de las diferencias en función de cada una de estas variables se realiza mediante el cálculo del correspondiente índice de significación, considerando apreciable el impacto únicamente cuando ese índice alcance un valor igual o inferior a .05. Así, aplicaremos la prueba t de Student para muestras independientes, entendiendo como variable de agrupación para muestras independientes la participación (o no) de los estudiantes en el proyecto PEINAS.

Tabla 2

Análisis comparativo de adquisición de competencias cívico-sociales

Item

$\begin{array}{cccccc}\begin{array}{c}\bar{x}_{1} \\ \text { Control }\end{array} & \begin{array}{c}\bar{x}_{2} \\ \text { Experi- } \\ \text { mental }\end{array} & \text { t } & \text { gl } & \text { sig } \\ 4.26 & 4.50 & -1.500 & 29.384 & .144\end{array}$

Soy capaz de trabajar de un modo

$\begin{array}{lllll}4.26 & 4.50 & -1.500 & 29.384 & .144\end{array}$

cooperativo con otras personas

Soy capaz de comunicarme adecua-

$\begin{array}{lllll}4.04 & 4.31 & -1.332 & 24.324 & .195\end{array}$

damente con los demás

Me relaciono fácilmente con otras

$\begin{array}{lllll}3.91 & 4.38 & -1.892 & 34.354 & .067\end{array}$

personas

Trato de ponerme en el lugar de los

$\begin{array}{lllll}4.52 & 4.81 & -1.983 & 36.323 & .055\end{array}$

demás para intentar comprender su

situación

Soy capaz de liderar grupos y mo-

$\begin{array}{lllll}3.87 & 3.88 & -.021 & 25.165 & .983\end{array}$

tivar a otros para conseguir metas

comunes

Tenemos que mirar más allá de las

$\begin{array}{lllll}4.61 & 4.94 & -2.709 & 34.208 & .010\end{array}$ personas para comprender sus problemas 
El aprendizaje-servicio como vía para el desarrollo de competencias interculturales en la Universidad

Alexandre Sotelino Losada, Miguel Ángel Santos Rego y Jesús García Álvarez

\begin{tabular}{|c|c|c|c|c|c|}
\hline Item & $\begin{array}{c}\overline{x_{1}} \\
\text { Control }\end{array}$ & $\begin{array}{l}\overline{\chi_{2}} \\
\text { Experi- } \\
\text { mental }\end{array}$ & $t$ & gl & sig \\
\hline $\begin{array}{l}\text { Necesitamos cambiar las actitudes } \\
\text { de la gente para solucionar los pro- } \\
\text { blemas sociales }\end{array}$ & 4.52 & 4.75 & -1.369 & 36.701 & .179 \\
\hline $\begin{array}{l}\text { Disfruto conociendo a personas } \\
\text { procedentes de contextos diferentes } \\
\text { al mío }\end{array}$ & 4.43 & 4.93 & -3.564 & 32.430 & .001 \\
\hline $\begin{array}{l}\text { La diversidad cultural hace a un gru- } \\
\text { po más interesante y efectivo }\end{array}$ & 4.74 & 5.00 & -2.787 & 22.000 & .011 \\
\hline $\begin{array}{l}\text { Me adapto con facilidad a otros am- } \\
\text { bientes culturales }\end{array}$ & 3.83 & 4.19 & -1.630 & 34.211 & .112 \\
\hline $\begin{array}{l}\text { En todo lo que hago, me esfuerzo } \\
\text { para ser una mejor persona }\end{array}$ & 4.52 & 4.50 & -.122 & 35.042 & .904 \\
\hline $\begin{array}{l}\text { Trato de asegurarme de que mis } \\
\text { acciones no dañen intencionalmente } \\
\text { a otra persona }\end{array}$ & 4.48 & 4.63 & -.835 & 35.528 & .410 \\
\hline $\begin{array}{l}\text { Cuando se trabaja en grupo, intento } \\
\text { asegurarme que todos sean escucha- } \\
\text { dos antes de tomar una decisión }\end{array}$ & 4.35 & 4.44 & -.334 & 27.611 & .741 \\
\hline $\begin{array}{l}\text { Creo que si todas las personas apren- } \\
\text { diesen y trabajasen cooperativamen- } \\
\text { te, muchos de los problemas de la } \\
\text { sociedad podrían solucionarse }\end{array}$ & 4.61 & 4.94 & -2.709 & 34.208 & .010 \\
\hline $\begin{array}{l}\text { Para lograr un trabajo, poseer buenas } \\
\text { competencias personales (responsa- } \\
\text { bilidad, honradez, etc.) es tan impor- } \\
\text { tante como tener buenas competen- } \\
\text { cias técnicas }\end{array}$ & 4.65 & 4.69 & -.167 & 35.525 & .868 \\
\hline $\begin{array}{l}\text { Me resulta fácil evaluar y aceptar las } \\
\text { consecuencias de mis decisiones }\end{array}$ & 3.87 & 3.81 & .286 & 28.549 & .777 \\
\hline $\begin{array}{l}\text { Soy capaz de identificar y controlar } \\
\text { mis emociones y las de los demás }\end{array}$ & 3.48 & 3.50 & -.080 & 28.034 & .937 \\
\hline $\begin{array}{l}\text { Soy capaz de exponer mis ideas y } \\
\text { puntos de vista con seguridad }\end{array}$ & 3.70 & 3.81 & -.353 & 30.872 & .727 \\
\hline $\begin{array}{l}\text { Soy capaz de generar nuevas ideas } \\
\text { (soluciones, productos, puntos de } \\
\text { vista...) }\end{array}$ & 4.13 & 4.44 & -1.495 & 23.200 & .148 \\
\hline $\begin{array}{l}\text { Soy capaz de analizar información } \\
\text { desde un punto de vista crítico }\end{array}$ & 4.26 & 4.50 & -.990 & 26.510 & .331 \\
\hline
\end{tabular}


Como podemos observar en la tabla (ver tabla 2 ) las medias de los participantes en el proyecto PEINAS $\left(\overline{x_{2}}\right)$ son ligeramente superiores a aquellos alumnos/as que no han tomado parte de esta experiencia de ApS $\left(\overline{x_{1}}\right)$. Incluso en algún caso observamos diferencias significativas entre ambos grupos.

Así, encontramos diferencias al cuestionar al alumnado sobre si es necesario mirar más allá de las personas para poder comprender sus problemas $(t=-2.1709 ; p=.010)$. Son los participantes del PEINAS los que muestran mayor grado de acuerdo en este sentido $\left(\overline{x_{2}}=4.94\right)$, mientras que los miembros del grupo control manifiestan mayor moderación en la respuesta $\left(\overline{x_{1}}=4.61\right)$. Sucede de igual modo cuando tienen que pronunciarse sobre si disfrutan conociendo a personas procedentes de contextos diferentes al propio. De nuevo el alumnado que ha participado en ApS es el que responde con un mayor grado de acuerdo $\left(\overline{x_{2}}=4.93\right)$, frente a aquellos que son menos receptivos en este sentido $\left(\overline{x_{1}}=4.43\right)$, dándose entre los grupos diferencias estadísticamente significativas ( $\mathrm{t}=-$ 3.564; $\mathrm{p}=.001$ ).

Existe también unanimidad en los implicados en el proyecto PEINAS cuando manifiestan que la diversidad cultural hace a un grupo más interesante y efectivo $\left(\overline{x_{2}}=5\right)$, al tiempo que sus compañeros del grupo control difieren en las opiniones $\left(\overline{x_{1}}=4.74\right)$, estableciendo diferencias entre los grupos ( $\mathrm{t}=-2.787, \mathrm{p}=.011)$. Se da un caso semejante cuando se pregunta al alumnado si cree que en el caso de que las personas aprendiesen y trabajasen cooperativamente, muchos de los problemas podrían solucionarse. De nuevo el alumnado de ApS obtiene una media superior $\left(\overline{x_{2}}=4.94\right)$ en sus respuestas, dando lugar a diferencias significativas ( $\mathrm{t}=-$ 2.709; $p=.010$ ) respecto a sus compañeros y compañeras $\left(\overline{x_{1}}=4.61\right)$ que no se implican en este tipo de actividades.

\subsection{Discusión de resultados}

Entendiendo los resultados en su totalidad cabe señalar que el alumnado de esta materia obtiene medias altas en las respuestas de la escala de competencias cívico-sociales (entre el 3 y el 5). Esto parece indicarnos, por un lado, que al cursar esta asignatura, sin olvidar la clara influencia del conjunto de la titulación, el alumnado adquiere una serie de competencias cívico-sociales, aspecto clave en la formación universitaria y en la convergencia europea (Gonzalez-Geraldo, Jover, y Martínez, 2017; 
Palos y Puig, 2015; Reparáz, Arbués, Naval, y Ugarte, 2015; Villa y Poblete, 2007). Y por otro, también parece claro que aquellos alumnos/ as que se han visto involucrados en el proyecto PEINAS desarrollan en mayor grado estos conocimientos, habilidades y actitudes, algo que se pone de manifiesto gracias a las altas puntuaciones medias señaladas en el cuestionario, y que en ciertos aspectos incluso marcan diferencias significativas con sus compañeros.

Por último, tampoco es baladí, que las variables donde se marcan mayores distancias entre los grupos y, por tanto, dan lugar a diferencias significativas, son aquellas que guardan estrecha relación con la temática de la materia, es decir, la pedagogía intercultural. Cabe prever, por lo tanto, que estableciendo un contacto directo con entidades que desarrollan su trabajo en el ámbito de la gestión de la diversidad cultural, y situando al alumno en relación con sus socios y usuarios, se desarrollan en mayor grado aquellas competencias que resultan básicas en la asignatura y a su vez están estrechamente vinculadas al ámbito profesional de la Pedagogía. Pero, además, no debemos descuidar el hecho, y así lo indican los resultados, de que hay aprendizajes personales y particulares que han arraigado en las alumnas y alumnos, toda vez que se han ido adquiriendo habilidades de corte relacional e intercultural.

\section{Conclusiones y prospectiva}

En este proyecto, junto con el aprendizaje de contenidos vinculados a la asignatura de Pedagogía Intercultural, hemos detectado un incremento de las expectativas personales y profesionales del alumnado en relación al desarrollo de competencias cívico-sociales, una mejora que valoramos positivamente a la vista de las habituales quejas de los estudiantes sobre la escasa conexión de la formación universitaria y el desempeño profesional (Santos-Rego y Lorenzo, 2007). A lo que añadimos los beneficios derivados del trabajo en red de los diferentes grupos y agentes sociales.

Los resultados obtenidos nos indican que los alumnos y alumnas que participan en un proyecto de aprendizaje-servicio como el que aquí se presenta, adquieren en mayor medida una serie de competencias y habilidades de corte cívico-social, y también relacional, que supondrán un aliciente en su formación profesional, sin olvidar el impacto que esto 
tendrá en su vida personal. Tratamos de poner de manifiesto que un mayor protagonismo del alumnado en sus procesos de aprendizaje implica una mejora de la Educación Superior. No puede extrañar, entonces, que en los últimos años aumentaran las voces que solicitan la introducción del aprendizaje-servicio en las aulas universitarias (Bringle y Hatcher, 1996; Furco, 2005; Lockeman y Pelco, 2013; Martínez, 2008; Rodríguez-Gallego, 2014; Sotelino et al., 2016).

Asimismo, algunos estudios que se han ocupado de analizar la inserción laboral de los titulados en Pedagogía, han demostrado que la implicación del alumnado en actividades de carácter experiencial contribuye, no solo a la búsqueda y acceso a un empleo, sino también a su posterior desarrollo profesional (ACSUG, 2016; ANECA, 2004; Ventura y Martínez, 2004). Es importante, de igual modo, en la medida en que ayudan a adquirir una determinada experiencia profesional, que el alumnado del título identifica como fundamental en su acceso al empleo pero que la universidad apenas le proporciona, tal y como muestra la investigación realizada por Jiménez (2007). Por ello, la institución universitaria se enfrenta hoy al reto de generar iniciativas dirigidas a promover y fomentar la planificación de actividades que supongan el contacto del alumnado con su entorno laboral más próximo, acercándolos a sus principales figuras profesionales y a un mercado laboral en constante evolución.

En este sentido, el aprendizaje-servicio se presenta como una oportunidad para que los estudiantes del Grado en Pedagogía, entre otras titulaciones en el amplio abanico de las Ciencias Sociales, puedan añadir un ingrediente a su formación. Este componente extra se debería traducir en un mejor desarrollo de competencias transversales, casi siempre asociadas a rutas de inserción laboral, pero que en muchas ocasiones aparecen diluidas en su formación.

Estamos convencidos de que los diferentes servicios realizados en las entidades anteriormente citadas supondrán aprendizajes significativos y valiosos para el alumnado en el marco de la Pedagogía, y por ende, de la Pedagogía Intercultural. El contacto con entidades que en su día a día trabajan en la gestión socioeducativa de la diversidad cultural, implica a los estudiantes en nuevas dinámicas organizativas con las que probablemente no están familiarizados. Por otro lado, tampoco podemos obviar el componente ético y moral del compromiso asumido, puesto que los participantes acaban por exponerse a nuevos puntos de vista y perspectivas que en ocasiones les colocan ante situaciones inusitadas, a 
El aprendizaje-servicio como vía para el desarrollo de competencias interculturales en la Universidad

Alexandre Sotelino losada, Miguel Ángel Santos Rego y Jesús García Álvarez

las cuales tendrán que dar cumplida respuesta. Ahí radica una parte sustantiva del interés pedagógico, toda vez que nuestros estudiantes habrán de participar también en la construcción de su propio aprendizaje, al tener que resolver problemas reales en contextos culturalmente diversos (Santos-Rego, Lorenzo, y Mella, 2016).

\section{Referencias}

ACSUG. (2016). Estudio de la inserción laboral de los titulados en el Sistema Universitario de Galicia 2011-2012. Santiago de Compostela: Autor.

Álvarez, J. L, Martínez, M. J., González, H. y Buenestado, M. (2017). El aprendizajeservicio en la formación del profesorado de las universidades españolas. Revista Española de Pedagogía, 267, 199-217.

ANECA. (2004). Libro Blanco. Título de Grado en Pedagogía y Educación Social. Madrid: Autor.

Boyle-Baise, M. (1998). Community Service Learning for Multicultural Education: An Exploratory Study with Preservice Teachers. Equity y Excellence in Education, 31(2), 52-60. doi: 10.1080/1066568980310207

Bringle, R. G. \& Hatcher, J. A. (1996). Implementing service learning in higher education. Journal of Higher Education, 67(2), 221-239. doi: 10.2307/2943981

Deeley, S. (2016). El aprendizaje-servicio en educación superior. Teoría, práctica y perspectiva crítica. Madrid: Narcea.

Driscoll, A., Holland, B., Gelmon, S. \& Kerrigan, S. (1996). An Assessment Model for Service-Learning: Comprehensive Case Studies of Impact on Faculty, Students, Community, and Institution. Michigan Journal of Community Service Learning, 3, 66-71.

Einfeld, A. \& Collins, D. (2008). The relationships between service-learning, social justice, multicultural competence, and civic engagement. Journal of College Student Development, 49(2), 95-109. doi: 10.1353/csd.2008.0017

Furco, A. (2005). Impacto de los proyectos de aprendizaje-servicio. En Programa Nacional Educación Solidaria, MECT, Aprendizaje y servicio solidario en la Educación Superior y en los sistemas educativos latinoamericanos (pp. 19-26). Buenos Aires: Actas del séptimo Seminario Internacional Aprendizaje y Servicio Solidario.

Goldberg, L. R. \& Coufal, K. L. (2009). Reflections on service-learning, critical thinking, and cultural competence. Journal of College Teaching y Learning, 6(6), 39-50.

González-Geraldo, J. L., Jover, G. y Martínez Martín, M. (2017). La ética del aprendizaje servicio en la Universidad: una interpretación desde el pragmatismo. Bordón, 69(4), 63-78. doi: 10.13042/Bordon.2017.690405

Jiménez, A. (2007). Aproximación a un diagnóstico comprensivo sobre la formación y la inserción laboral en la titulación de Pedagogía de la UPSA. Papeles Salmantinos de Educación, 8, 95-117.

Lockeman, K. \& Pelco, L. E. (2013). The Relationship between Service-Learning and Degree Completion. Michigan Journal of Community Service-Learning, 20(1), 18-30. 
El aprendizaje-servicio como vía para el desarrollo de competencias interculturales en

Lorenzo, M., Mella, I., García, J. y Varela, C. (2017). Investigar para institucionalizar el aprendizaje servicio en la universidad española. RIDAS, Revista Iberoamericana de Aprendizaje Servicio, 3, 118-130. doi: 10.1344/RIDAS2017.3.9

Lorenzo, M. y Santos Rego, M. A. (2009). Educación para a cidadanía e os profesores. Visión e desafío. Vigo: Edicións Xerais.

Martínez, M. (Ed.). (2008). Aprendizaje servicio y responsabilidad social de las universidades. Barcelona: Octaedro.

Martínez-Usarralde, M. J. (2009). Educación Internacional. Valencia: Tirant lo Blanch.

Martínez-Vivot, M. y Folgueiras, P. (2015). Evaluación participativa, aprendizaje-servicio y Universidad. Profesorado, revista de currículum y formación del profesorado, 19(1), 128-143.

O'Grady, C. R. (2000). Integrating service learning and multicultural education in colleges and universities. Mahwah, New Jersey: L. Erlbaum Associates.

Paéz, M. y Puig, J. M. (2013). La reflexión en el aprendizaje-servicio. Revista internacional de educación para la justicia social (RIEJS), 2(2), 13-32.

Palos, J. y Puig, J. (2015). Las competencias son el foco de la evaluación del alumnado en los proyectos de aprendizaje servicio. En J. Puig (Coord.), ¿Cómo realizar un proyecto de aprendizaje servicio?: 11 ideas clave (pp. 115-130). Barcelona: Graó.

Priegue, D. y Sotelino, A. (2016). Aprendizaje-servicio y construcción de una ciudadanía intercultural: El proyecto PEINAS. Foro de Educación, 14(20), 361-382.

Reparáz, C., Arbués, E., Naval, C. y Ugarte, C. (2015). El Índice Cívico de los universitarios sus conocimientos, actitudes y habilidades de participación social. Revista Española de Pedagogía, 73(260), 23-52.

Rodríguez-Gallego, M. R. (2014) El aprendizaje-servicio como estrategia metodológica en la Universidad. Revista Complutense de Educación, 25(1), 95-113. doi: 10.5209/ rev_rced.2014.v25.n1.41157

Santos-Rego, M. A., Jover, G., Naval, C., Álvarez, J. L., Vázquez, V. y Sotelino, A. (2017). Diseño y validación de un cuestionario sobre práctica docente y actitud del profesorado universitario hacia la innovación (CUPAIN). Educación XX1, 20(2), 39-71, doi: 10.5944/educXX1.17806

Santos Rego, M. A., Sotelino, A. y Lorenzo, M. (2015). Aprendizaje-servicio y misión cívica de la universidad. Una propuesta de desarrollo. Barcelona: Octaedro.

Santos-Rego, M. A. y Lorenzo, M. (2007). Universidade e construcción da sociedade civil. Vigo: Xerais.

Santos-Rego, M. A. (2013). ¿Para cuándo las universidades en la agenda de una democracia fuerte? educación, aprendizaje y compromiso cívico en Norteamérica. Revista de educación, 361, 565-590.

Santos-Rego, M. A. (2017). La educación intercultural y el pluralismo religioso. Propuestas pedagógicas para el diálogo. Educación XX1: Revista de la Facultad de Educación, 20(1), 17-35.

Santos-Rego, M. A., Lorenzo, M. y Mella, I. (2016). Aprendizaje-servicio y desempeño académico de los estudiantes universitarios. En M. A. Santos-Rego (Ed.), Sociedad del conocimiento. Aprendizaje e Innovación en la Universidad (pp. 197-218). Madrid: Biblioteca Nueva. 
El aprendizaje-servicio como vía para el desarrollo de competencias interculturales en la Universidad

Alexandre Sotelino losada, Miguel Ángel Santos Rego y Jesús García Álvarez

Santos-Rego, M. A. y Lorenzo, M. (2012). Estudios de Pedagogía Intercultural. Barcelona: Octaedro.

Sotelino, A. (2014). Pedagogía intercultural y aprendizaje-servicio. El proyecto peinas. En L. Rubio, L. Moliner y A. Francisco, Construyendo ciudadanía crítica y activa: Experiencias sobre el aprendizaje-servicio en las universidades del Estado español (pp. 61-78). Barcelona: Icaria.

Sotelino, A., Santos Rego, M. A. y Lorenzo, M. (2016). Aprender y Servir en la Universidad: Una vía cívica al desarrollo educativo. Teoría de la Educación, 28(2), 225-228. doi: 10.14201/teoredu282225248

Tejada, J. (2013). La formación de las competencias profesionales a través del aprendizaje-servicio. Cultura y Educación: Culture and Education, 25(3), 285-294.

Ventura, J. y Martínez Olmo, F. (2004). Estudio sobre la inserción laboral de los graduados en Pedagogía en la Universidad de Barcelona. Barcelona: Universidad de Barcelona.

Villa, A. y Poblete, M. (Dirs.). (2007). Aprendizaje basado en competencias. Una propuesta para la evaluación de las competencias genéricas. Bilbao: Mensajero/ICE Universidad de Deusto. 